\title{
Effect of oral supplementation with enzymatically synthesized glycogen (ESG) on cognitive function: a randomized, double-blind, placebo-controlled study
}

\section{Takashi Furuyashiki, Ryo Kakutani*, Mayumi Hokoyama-Fujita, Kazuko Kato, Hiroshi Kamasaka and Takashi Kuriki}

Institute of Health Sciences, Ezaki Glico Co., Ltd., Nishiyodogawa-ku, Osaka, Japan, 555-8502, Japan

*Corresponding author: Ryo Kakutani, PhD, Institute of Health Sciences, Ezaki Glico Co., Ltd. 4-6-5, Utajima, Nishiyodogawa-ku, Osaka 555-8502, Japan

Submission Date: March $2^{\text {nd }}$, 2020; Acceptance Date: April 27 $7^{\text {th }}$ 2020; Publication Date: April $30^{\text {th }}, 2020$

Citation: Takashi Furuyashiki T., Kakutani R, Hokoyama-Fujita M., Kato K., Kamasaka H., and Takashi Kuriki T. Effect of oral supplementation with enzymatically synthesized glycogen (ESG) on cognitive function: a randomized, double-blind, placebo-controlled study. Functional Foods in Health and Disease. 2020; 10(4): 155-167. DOI: https://doi.org/10.31989/ffhd.v10i4.700

This article is a part of special issue of ICoFF/ISNFF 2019, Kobe, Japan. Special issue editors: Yasuhito Shirai, PhD, Professor, Graduate School of Agricultural Science, Department of Agrobioscience, Kobe University, Kobe, Japan and Hiroshi Yoshida, MD, PhD, Professor, The Jikei University School of Medicine, Tokyo, Japan

\begin{abstract}
Background: There is a well-established correlation between aging and decreasing cognitive performance in healthy adults. Furthermore, with increasing levels of stress in modern societies, cognitive decline is a growing concern. With our focus on these concens, we prepared enzymatically synthesized glycogen (ESG) from starch, and aimed to examine whether ESG supplementation improved cognitive functions in humans.
\end{abstract}

Methods: In a randomized, double-blind, placebo-controlled, cross-over trial, 40 healthy participants were administered $5.0 \mathrm{~g}$ of ESG or maltodextrin (placebo) beverages for 4 weeks, respectively. A washout period of 4 to 5 weeks was set between treatments. The primary endpoint 
was the effect of orally administered ESG on cognitive function, which was assessed by using the CogHealth test battery. In addition, the fatigue VAS (visual analog scale) score and salivary levels of anti-fatigue factors (such as cortisol and secretory $\operatorname{IgA}$ ) were determined.

Results: Two participants dropped out for personal reasons, therefore data for the remaining 38 subjects was analyzed. It was found that visual discrimination and long-term memory were significantly potentiated by the ingestion of ESG for 2-4 weeks compared with placebo treatment. On the other hand, the fatigue VAS score and salivary levels of anti-fatigue factors showed no significant differences between the ESG group and the placebo group.

Conclusions: Our study shows that oral administration of ESG significantly potentiates the cognitive performance of healthy volunteers. We speculate that glycogen is not only a vital energy source, but is also involved in enhancement of cognitive function.

Keywords: Glycogen, ESG, cognitive performance, CogHealth, long-term memory, visual discrimination.

\section{INTRODUCTION}

Glycogen is a multibranched polysaccharide in which glucose molecules are connected by a-1,4 glycoside bonds and the branches are connected by a-1,6 glycoside bonds. Its molecular weight ranges from $10^{6}$ to $10^{9}$ and it is a spherical nanoparticle with a diameter of 20-60 nm. Glycogen is found widely in nature, and is the major storage polysaccharide in animals, shellfish, and microorganisms [1]. In mammals, the largest glycogen reserves exist in the liver and skeletal muscles. The major functions of glycogen are to supply energy to the muscles and to release glucose into the blood from the liver [1]. Small quantities of glycogen are also present in other tissues, such as the brain, thymus, heart, skin, placenta, and leukocytes. It is unclear whether glycogen has other functions besides acting as an energy source [2-7].

In 1998, it was reported that glycogen extracted from scallops and oysters possessed immunomodulatory activity, while other glycogen preparations had little or no activity [8]. The authors suggested that these differing effects of glycogen might result from fine structural differences, which could depend on many factors such as the method of extraction or the source. To overcome the influence of such factors, we developed methods for enzymatic synthesis of homogeneous glycogen preparations [9]. These methods allow us to control several parameters of glycogen, such as the fine structure, molecular weight, and molecular size. Our enzymatically synthesized glycogen (ESG) has the same characteristics as glycogen from natural sources [10,11]. Using ESG, we have obtained the following findings: (i) glycogen activates macrophages, which 
are important immune cells involved in antigen recognition, to produce nitric oxide and inflammatory cytokines such as interleukin-6 or interleukin-12 [12, 13], (ii) Toll-like receptor 2, which is a receptor involved in innate immunity, mediates the response of macrophages to glycogen [14], and (iii) orally administered ESG enhances systemic immune responses such as a tumor suppression effect, activation of natural killer cells in the spleen, and suppression of splenic sympathetic nervous activity [15]. These results suggest that glycogen not only has a role in maintaining blood glucose levels, but also has immunomodulatory effects. Moreover, it has been reported that orally administered ESG has prebiotic effects, including the improvement of lipid metabolism and modulation of enteric bacterial flora [16, 17]. Thus, various effects of orally administered ESG have been demonstrated.

It is well known that cognitive function gradually decreases with aging, while it declines rapidly in patients with dementia, such as Alzheimer's disease [18]. In addition, cognitive performance is decreased by alcohol intake, acute and chronic fatigue, and stress [19, 20]. Cognitive deficits in areas such as attention, memory, and reaction time significantly reduce one's quality of life. Therefore, improvement of cognitive performance is the target of much research. It has been reported that astaxanthin and flavonoids can improve age-related cognitive decline. For example, administration of astaxanthin for 12 weeks significantly improved the cognitive performance of elderly subjects, who complained of age-related forgetfulness [21]. These subjects performed the CogHealth test, which is designed to evaluate cognitive performance by playing card games on a personal computer and can detect slight changes in healthy or mildly impaired subjects at an early stage of decline [21,22]. It was also reported that a flavonoid-rich extract of Pinus radiata bark improves cognitive function by enhancing immediate recognition and spatial working memory [23]. These reports suggest that oral administration of certain food components may enhance cognitive function. Accordingly, we examined whether the cognitive performance of healthy subjects was enhanced specifically by the oral administration of ESG. Using a doubleblind, randomized, placebo-controlled, cross-over, study design we found that oral administration of ESG for 2-4 weeks significantly potentiated the cognitive performance of healthy volunteers. This included reaction times in the "choice reaction task" and accuracy in the "one card learning task". To the best of our knowledge, this is the first study investigating the effect of orally administered glycogen on cognitive brain function in humans. Our findings suggest that glycogen can potentially be used as a food component that improves cognitive function.

\section{MATERIALS AND METHODS}

\section{Preparation of ESG}

ESG (Bioglycogen ${ }^{\mathrm{TM}}$; Glico Nutrition Co., Ltd.) was prepared as described previously [9]. The a-1,6-glucosidic linkages of starch were completely digested with isoamylase which was derived 
from Pseudomonas amyloderamosa to produce short chain amylose. After heating to inactivate the enzyme, recombinant amylomaltase, derived from Thermus aquaticus and the branching enzyme from Aquifex aeolicus, were added to the reaction mixture [24,25]. Since the A. aeolicus branching enzyme does not react efficiently with short chain amylose, amylomaltase was employed to elongate amylose and increase the efficiency of the branching reaction, allowing ESG to be formed from elongated amylose. In the present study, ESG was administered as a beverage. The composition of the ESG beverage and placebo beverage are shown in Table 1. Fifty milliliters of the ESG beverage contained $5.0 \mathrm{~g}$ of ESG, $15 \mathrm{mg}$ of citric acid, and $3.0 \mathrm{mg}$ of sucralose, while the placebo beverage contained $15 \mathrm{mg}$ of citric acid, $3.0 \mathrm{mg}$ of sucralose, and $5.0 \mathrm{~g}$ dextrin (a1,4/1,6 glucans) in place of the ESG. For blinding, all beverages were sealed in identical aluminum bottles and sterilized. It was impossible to discriminate between the ESG and placebo beverages in terms of taste and appearance.

Table 1. Composition of the placebo and ESG beverages

\begin{tabular}{lrr}
\hline & $\begin{array}{r}\text { Placebo } \\
\text { beverage }\end{array}$ & $\begin{array}{r}\text { ESG } \\
\text { beverage }\end{array}$ \\
\hline ESG (mg) & 0 & 5000 \\
Maltodextrin (mg) & 5000 & 0 \\
Sucralose (mg) & 3.0 & 3.0 \\
Citric acid (mg) & 15 & 15 \\
Total volume (ml) & 50 & 50 \\
\hline Energy (kcal) & 20 & 20 \\
Protein (g) & 0.05 & $<0.05$ \\
Lipid (g) & $<0.05$ & $<0.05$ \\
Carbohydrate $(\mathrm{g})$ & 5.0 & 5.0 \\
Sodium $(\mathrm{mg})$ & 0 & 0 \\
\hline
\end{tabular}

*Each beverage was ingested once daily $(50 \mathrm{~mL})$.

\section{Subjects}

Forty healthy adults (25 men and 15 women) aged between 25 and 58 years participated in this study. All subjects were employed full-time, and worked approximately $7.5 \mathrm{~h} /$ day, 5 days/week. Since 2 participants dropped out for personal reasons, data on the remaining 38 subjects was used for analysis. The mean \pm SD age, height, weight, and body mass index of these 38 subjects (23 men and 15 women) was $41.8 \pm 8.03$ years $(45.0 \pm 7.40$ years for men and $36.9 \pm 6.50$ years for women $), 164.0 \pm 8.58 \mathrm{~cm}(169.4 \pm 6.05 \mathrm{~cm}$ and $155.9 \pm 4.39 \mathrm{~cm}), 61.0 \pm 11.2 \mathrm{~kg}(66.0 \pm 8.33 \mathrm{~kg}$ and $53.5 \pm 11.1 \mathrm{~kg})$, and $22.6 \pm 3.39 \mathrm{~kg} / \mathrm{m}^{2}\left(23.0 \pm 2.97 \mathrm{~kg} / \mathrm{m}^{2}\right.$ and $\left.22.0 \pm 3.96 \mathrm{~kg} / \mathrm{m}^{2}\right)$, respectively (Table 2). 
Table 2. Baseline characteristics of the subjects

\begin{tabular}{|c|c|c|c|c|c|c|}
\hline \multirow[b]{3}{*}{ Characteristic } & \multicolumn{6}{|c|}{ Subjects } \\
\hline & \multicolumn{2}{|c|}{ All $(n=38)$} & \multicolumn{2}{|c|}{$\operatorname{Men}(n=23)$} & \multicolumn{2}{|c|}{ Women $(n=15)$} \\
\hline & Mean & SD & Mean & $\mathrm{SD}$ & Mean & $\mathrm{SD}$ \\
\hline Age (y) & 41.8 & 8.03 & 45.0 & 7.40 & 36.9 & 6.50 \\
\hline Height (cm) & 164.0 & 8.58 & 169.4 & 6.05 & 155.9 & 4.39 \\
\hline Weight (kg) & 61.1 & 11.2 & 66.0 & 8.33 & 53.5 & 11.1 \\
\hline BMI $\left(\mathrm{kg} / \mathrm{m}^{2}\right)$ & 22.6 & 3.39 & 23.0 & 2.97 & 22.0 & 3.96 \\
\hline
\end{tabular}

*Two dropouts were excluded from this analysis.

This study was conducted from November 2013 to February 2014. The study protocol and the informed consent document conformed to the guidelines of the Declaration of Helsinki, and were approved by the ethics committee of Ezaki Glico Co., Ltd (Registration Number: 2013-7). All participants provided written informed consent prior to participation.

\section{Study design}

This was a randomized, double-blind, placebo-controlled, cross-over study (Fig. 1).

\begin{tabular}{|c|c|}
\hline \multicolumn{2}{|c|}{40 healthy volunteers randomly allocated to Group A or Group B } \\
\hline $\begin{array}{l}\text { Group A ( } n=20) \\
\text { Placebo beverage for } 4 \text { weeks }\end{array}$ & $\begin{array}{l}\text { Group B }(n=20) \\
\text { ESG beverage for } 4 \text { weeks }\end{array}$ \\
\hline \multicolumn{2}{|c|}{$\begin{array}{l}\text { Before (baseline) and after } 2 \text { and } 4 \text { weeks of beverage intake, } \\
\text { sampling of saliva, cognitive function tests, and the fatigue VAS } \\
\text { were performed }\end{array}$} \\
\hline & Washout period of $4-5$ weeks \\
\hline $\begin{array}{l}\text { Group A ( } n=20) \\
\text { ESG beverage for } 4 \text { weeks }\end{array}$ & $\begin{array}{l}\text { Group B }(n=20) \\
\text { Placebo beverage for } 4 \text { weeks }\end{array}$ \\
\hline & 2 dropouts \\
\hline \multicolumn{2}{|c|}{$\begin{array}{l}\text { Before (baseline) and after } 2 \text { and } 4 \text { weeks of beverage intake, } \\
\text { sampling of saliva, cognitive function tests, and the fatigue VAS } \\
\text { were repeated }\end{array}$} \\
\hline
\end{tabular}

Fig.1 Flow diagram of the study 
The primary endpoint was the effect of orally administered ESG on cognitive function which was assessed by using the CogHealth test battery. Thirty-eight subjects were randomly divided into two groups (group A and group B). All of the subjects, study administrators, and data processors were completely blind to the assignment of treatment groups.

During the first experimental period, the subjects ingested the assigned beverages once daily between 13:00 and 18:00 for 4 weeks; group A ingested the ESG beverage, while group B ingested the placebo beverage. After a washout period of 4 to 5 weeks, the beverages were reversed in the second experimental period. In each experimental period, cognitive testing and saliva sampling was completed three times; before treatment (baseline), after 2 weeks, and after 4 weeks of ingestion.

Prior to conducting the tests, subjects ate a meal of Japanese noodles between 12:00 - 12:30, after which any oral intake other than water was prohibited until 16:00. Subjects entered the test room at 16:00 and their level of fatigue was assessed using a simple horizontal visual analog scale (VAS), followed by the cognitive test for about $5 \mathrm{~min}$. Subjects were allowed to rest for 20 minutes while sitting in a chair. A saliva sample was collected using a Salivette (Sartrious Co.) at 16:25, and cognitive testing began again at 16:30. In order to prevent influence of the ingested beverage on the testing day, each subject ingested ESG or placebo beverage after finishing the cognitive testing.

\section{Cognitive testing}

Cognitive performance was assessed using the CogHealth test. The battery of tests consist of four tasks: "simple reaction task", "choice reaction task", "working memory task" (short-term working memory), and "one card learning task" (delayed recall).

For the "simple reaction task", a card is presented face down in the center of the computer screen. When this card turns face up, participants are required to press the "k" key as quickly as possible. There are 35 trials per task, and the first task is repeated again after the one card learning task (the last task).

The "choice reaction task" is similar to the "simple reaction task". Participants are required to indicate the color of the card suit (black or red) by pressing the "d" key or the " $\mathrm{k}$ " key depending on the color. This task is continued until the participant has made 30 correct responses or until the maximum time is reached.

The "working memory task" assesses short-term memory. A single card is presented in the center of the screen and it changes every 2 seconds. Each time the card changes, the participant must indicate whether or not the new card is the same as the previous card by pressing either the " $\mathrm{k}$ " key or the " $\mathrm{d}$ " key. When the response is correct, the card is moved to a single pack. This task is continued until the participant has made 30 correct responses or until the maximum time is reached.

The "one card learning task" is performed to assess delayed recall. A single card is presented in the center of the screen and it changes every 2 seconds. Each time the card changes, the 
participant must indicate whether or not the new card has already been presented in this task by pressing either the " $\mathrm{k}$ " key or the " $\mathrm{d}$ " key. When the response is correct, the card is moved to a single pack. This task is continued until the participant has made 42 correct responses or until the maximum time is reached.

To minimize the learning effect, all subjects are trained in performing the CogHealth battery of tests for one week before the trial.

\section{Assessment of fatigue}

The evaluation with VAS was carried out as soon as the subject entered the test room at 16:00. Subjects were instructed to make a mark on the fatigue VAS, which was a straight horizontal line $100 \mathrm{~mm}$ in length, according to their fatigue level. Results were expressed in millimeters from zero (no fatigue: the left end of the line) to 100 (severe fatigue: the right end of the line).

\section{Collection and analysis of saliva}

Before ingestion of ESG (baseline) and after 2 and 4 weeks of ESG intake, saliva was collected from all subjects. A cotton Salivette (Sartrious Co.) was placed in the oral cavity and chewed for 3 minutes, after which a saliva sample was obtained by centrifugation of the retrieved Salivette. All saliva samples were stored at $-80^{\circ} \mathrm{C}$ until analysis.

Levels of fatigue-related factors in saliva samples were measured by using assay kits. Secretory $\operatorname{IgA}$ (sIgA) and cortisol concentrations were determined with the sIgA Salivary EIA Kit and Cortisol Salivary EIA Kit (Salimetrics Co.), while a-amylase activity was measured with an assay from Oriental Yeast Co., Ltd..

\section{Statistical analysis}

All analyses were performed using SPSS 16.0J software for Windows (SPSS Japan Inc., Tokyo, Japan). The significance of differences between the ESG group and the placebo group was determined with the paired $t$-test. Differences between baseline parameters and those obtained at later times during the study were analyzed by using Dunnett's multiple comparison test. In all analyses, $p<0.05$ was considered significant.

\section{RESULTS}

\section{Disposition of the subjects}

We recruited 40 subjects and 38 of them completed this trial. The other two subjects dropped out for personal reasons unrelated to ingestion of the ESG and placebo beverages.

\section{Effect of ESG on cognitive performance}

Data on cognitive performance (CogHealth battery) are shown in Table 3 as relative values compared with the baseline results. Reaction times in the "simple reaction task" and "working memory task" were significantly shorter after 4 weeks of ESG beverage intake compared with 
baseline, while no such effect was observed with the placebo beverage. Also, the reaction time in the "choice reaction task" and accuracy in the "one card learning task" were significantly improved after 2 weeks and 4 weeks of ESG beverage intake, respectively, compared with the results for the placebo beverage. These findings suggest that ESG intake could improve several aspects of cognitive performance such as visual discrimination and memory.

Table 3. Relative mean reaction times and accuracy in the CogHealth test battery at baseline and after 2 and 4 weeks of ESG intake.

\begin{tabular}{lllll}
\hline Baseline & 2 weeks & 4weeks & \\
\cline { 2 - 3 }$\pm \mathrm{SD}$ & Mean \pm SD & $\begin{array}{l}p \text { value } \\
\text { (vs Placebo) }\end{array}$ & Mean \pm SD & $\begin{array}{l}p \text { value } \\
\text { (vs Placebo) }\end{array}$ \\
\hline
\end{tabular}

Simple reaction task: reaction time

\begin{tabular}{|c|c|c|c|c|}
\hline Placebo & $100.0 \pm 15.35$ & $99.10 \pm 12.91$ & & $94.61 \pm 21.68$ \\
\hline ESG & $100.0 \pm 17.91$ & $98.16 \pm 13.42$ & 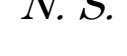 & $94.10 \pm 20.16$ \\
\hline
\end{tabular}

Choice reaction task: reaction time

\begin{tabular}{|c|c|c|c|c|}
\hline Placebo & $100.0 \pm 14.93$ & $101.6 \pm 14.14$ & \multirow{2}{*}{$p<0.05$} & $99.58 \pm 16.21$ \\
\hline ESG & $100.0 \pm 18.08$ & $97.81 \pm 13.21$ & & $101.4 \pm 21.47$ \\
\hline
\end{tabular}

Working memory task: reaction time

\begin{tabular}{|c|c|c|c|c|}
\hline Placebo & $100.0 \pm 18.25$ & $97.58 \pm 15.83$ & & $96.50 \pm 17.96$ \\
\hline ESG & $100.0 \pm 17.26$ & $95.63 \pm 14.84 *$ & IV. & $94.76 \pm 17.87$ \\
\hline
\end{tabular}

One card learning task: reaction time

\begin{tabular}{|c|c|c|c|c|}
\hline Placebo & $100.0 \pm 39.58$ & $97.36 \pm 26.24$ & & $95.07 \pm 24.54$ \\
\hline ESG & $100.0 \pm 21.98$ & $98.27 \pm 26.41$ & 2.0 & $97.36 \pm 23.76$ \\
\hline
\end{tabular}

Working memory task: accuracy

\begin{tabular}{|c|c|c|c|c|}
\hline Placebo & $100.0 \pm 5.624$ & $100.9 \pm 4.513$ & & $99.40 \pm 5.822$ \\
\hline ESG & $100.0 \pm 4.176$ & $99.51 \pm 3.543$ & & $99.84 \pm 3.112$ \\
\hline
\end{tabular}

One card learning task: accuracy

\begin{tabular}{|c|c|c|c|c|c|}
\hline Placebo & $100.0 \pm 14.74$ & $99.58 \pm 13.41$ & 7 & $99.42 \pm 13.90$ & $\neg$ \\
\hline ESG & $100.0 \pm 14.66$ & $101.8 \pm 13.48$ & & $104.5 \pm 15.33$ & \\
\hline
\end{tabular}

Two dropouts were excluded from data analysis. *: $p<0.05$ vs. baseline. N.S.: not significant $v s$. placebo group. Data after 2 and 4 weeks of ESG intake are relative value compared to baseline. Differences between the ESG group and placebo group were determined with the paired $t$-test. Multiple comparisons between 2 or 4 weeks and baseline were performed using Dunnett's test. 


\section{Effect of ESG on fatigue and fatigue-related factors in saliva}

Table 4 shows the results obtained by measuring several fatigue-related factors in saliva and the VAS fatigue scores. Salivary levels of a-amylase activity, cortisol, and sIgA showed no significant differences between ingestion of the ESG and placebo beverages. Similarly, there was no significant difference of the VAS fatigue scores between the ESG and placebo beverages. Therefore, these results suggest that ingestion of ESG did not affect fatigue during daily life.

Table 4. Mean fatigue VAS score and salivary parameters at baseline and after ESG ingestion

\begin{tabular}{|c|c|c|c|c|c|}
\hline & Baseline & 2 weeks & & weeks & \\
\hline & Mean \pm SD & Mean \pm SD & $\begin{array}{c}p \text { value } \\
\text { (vs Placebo) }\end{array}$ & Mean \pm SD & $\begin{array}{c}p \text { value } \\
\text { (vs Placebo) }\end{array}$ \\
\hline \multicolumn{6}{|c|}{ Fatigue VAS (mm) } \\
\hline Placebo & $52.46 \pm 19.65$ & $51.62 \pm 18.84$ & \multirow{2}{*}{ N. S. } & $52.45 \pm 15.67$ & \multirow{2}{*}{$N . S$} \\
\hline ESG & $53.83 \pm 16.84$ & $50.51 \pm 18.51$ & & $52.99 \pm 18.05$ & \\
\hline \multicolumn{6}{|c|}{$\operatorname{slg} \mathrm{A}(\mu \mathrm{g} / \mathrm{ml})$} \\
\hline Placebo & $23.03 \pm 14.38$ & $25.10 \pm 14.19$ & \multirow{2}{*}{ N. S. } & $23.02 \pm 12.11$ & \multirow{2}{*}{$N . S$} \\
\hline ESG & $23.59 \pm 17.69$ & $25.46 \pm 16.33$ & & $20.47 \pm 17.44$ & \\
\hline \multicolumn{6}{|l|}{$\alpha$-Amylase } \\
\hline \multicolumn{6}{|c|}{ activity (IU/ml) } \\
\hline Placebo & $200.7 \pm 134.9$ & $213.1 \pm 140.4$ & \multirow{2}{*}{$N . S$. } & $239.0 \pm 156.4 *$ & \multirow{2}{*}{$N . S$} \\
\hline ESG & $204.5 \pm 134.5$ & $225.4 \pm 151.2$ & & $244.1 \pm 179.2 *$ & \\
\hline \multicolumn{6}{|c|}{ Cortisol (ng/dl) } \\
\hline Placebo & $94.07 \pm 51.95$ & $134.8 \pm 105.0 * *$ & \multirow{2}{*}{ N. $S}$. & $123.8 \pm 70.43 * *$ & \multirow{2}{*}{$N . S}$. \\
\hline ESG & $89.91 \pm 64.21$ & $115.6 \pm 50.29 * *$ & & $130.4 \pm 64.93 * *$ & \\
\hline
\end{tabular}

Two dropouts were excluded from data analysis. *: $p<0.05$ vs. baseline. $* *: p<0.01$ vs. baseline. N.S.: not significant vs. placebo group. Differences between the ESG group and placebo group were determined with the paired $t$-test. Multiple comparisons between 2 or 4 weeks and baseline were performed using Dunnett's test.

\section{DISCUSSION}

This randomized cross-over study was performed to investigate whether ESG enhanced cognitive performance, revealing that cognitive parameters such as visual discrimination and memory, assessed by CogHealth battery tests, were significantly improved by intake of ESG compared with 
placebo (Table 3). Although, both ESG and the placebo used in this study (dextrin) are both $(1 \rightarrow 4)(1 \rightarrow 6)$-linked a-D-glucans, ESG contains far more a-1,6 branches than dextrin and is a high-density spherical molecule [11]. These features of ESG may result in minimal penetration of digestive enzymes such as a-amylase and a-glucosidase, resulting in enzymes gradually digesting ESG molecules from the periphery. Since enzymatic degradation of ESG is much slower than for dextrin, ESG may reach the distal small intestine [15, 17, 26]. It is possible that this feature of ESG is important for exerting its functions on digestive organs such as the small intestine after oral administration. Since ESG is a form of glycogen produced from starch, it has very low toxicity and the oral $\mathrm{LD}_{50}$ is $>2000 \mathrm{mg} / \mathrm{kg} /$ day [27], suggesting that it can potentially be used as a new food ingredient for improvement of cognitive performance.

It is common knowledge that cognitive performance is impaired by alcohol intake and by fatigue related to sleep disruption [19, 20]. Falleti and Maruff et al. both reported that the blood alcohol concentration of $0.05 \%$ or sustained wakefulness for $24 \mathrm{~h}$ could be similarly compared in the performance of cognitive function [19]. These findings suggest that controlling fatigue could induce the improvement of cognitive performance. To investigate whether improvement of fatigue was relevant to the cognitive effect of ESG, we examined salivary levels of sIgA, cortisol, aamylase, and the VAS fatigue score before the start of ESG intake and after ESG ingestion for 2 and 4 weeks. However, we found no significant difference of the salivary fatigue-related factors between subjects taking ESG and subjects taking placebo (Table 4). VAS scores also showed no significant difference between ESG and placebo. These findings indicated that the mechanism by which ESG improves cognitive performance is unrelated to an effect on fatigue.

A possible underlying mechanism on the effects of ESG may involve activation of the immune system. It has been reported that deterioration of cognitive function is accelerated by age-related immunodeficiency, with the learning performance of mice showing significant impairment10 months after resection of the thymus [28]. In addition, the immune response of splenocytes is markedly reduced by aging in senescence accelerated mouse-prone 8 (SAMP8) mice. Furthermore, Lal et al. reported that immune senescence can induce deterioration of cognitive function [29]. They irradiated young mice to inactivate their immune systems, and then injected spleen and bone marrow cells obtained from aged mice with impaired learning, or cells from normal young mice. After injection of cells from aged mice, the young mice showed similar learning deficits to aged animals, while young mice receiving cells harvested from other young mice exhibited normal learning. These findings suggest that a healthy immune system is involved in the maintenance of normal cognitive function. Our previous studies have shown that oral ESG ingestion activates the immune system [15]. For example, we found that oral administration of ESG to mice activated $\mathrm{CD} 11 \mathrm{~b}^{+} \mathrm{CD} 11 \mathrm{c}^{+}$cells, such as macrophages and dendritic cells, in Peyer's patches, which are collections of lymphoid tissue in the small intestinal mucosa with an important role in the immune 
response. In addition, splenic natural killer cells were activated by ESG treatment, while splenic sympathetic nervous activity was suppressed. Moreover, ingestion of ESG suppressed tumor proliferation in mice and prolonged survival [15]. Thus, our findings suggest that oral intake of ESG induces systemic immune activation. Such activation of the immune system by ESG may be related to improvement of cognitive performance in the present study. Investigations to clarify the mechanism by which ESG alters cognitive function are required in the future.

Acknowledgments: We thank Ms. Akiko Fujiwara (Health Solution, Inc.) for her advice with CogHealth data analysis.

Competing interests: The authors are employees of Ezaki Glico Co., Ltd., however, this does not alter the author's adherence to the journal's policy.

Authors' contributions: All authors contributed as follows: Takashi Furuyashiki and Ryo Kakutani designed, carried out the experiments and co-wrote the manuscript. Mayumi HokoyamaFujita carried out the experiments. Kazuko Kato co-wrote the manuscript. Hiroshi Kamasaka and Takashi Kuriki gave advice in discussion and proofread the manuscript.

\section{REFERENCES:}

1. Geddes R. Glycogen: a metabolic viewpoint. Biosci Rep 1986, 6:415-428.

2. Brown AM. Brain glycogen re-awakened. J Neurochem 2004, 89:537-552.

3. Salmoral EM, Tolmasky DS, Krisman CR. Evidence for the presence of glycogen in rat thymus. Cell Mol Biol 1990, 36:163-174.

4. Taegtmeyer H. Glycogen in the heart - an expanded view. J Mol Cell Card 2004, 37:710.

5. Harmon CS, Phizackerley PJR. The effects of starvation and re-feeding on glycogen metabolism in mouse tail skin. Biochem J 1983, 212:679-683.

6. Blows JMH, Calder PC, Geddes R, Wills PR. The structure of placental glycogen. Placenta 1988, 9:493-500.

7. Scott RB, Still WJ. Glycogen in human peripheral blood leukocytes. II. The macromolecular state of leukocyte glycogen. J Clin Invest 1968, 47:353-359.

8. Takaya Y, Uchisawa H, Ichinohe H, Sasaki J, Ishida K, Matsue H. Antitumor glycogen from scallops and the interrelationship of structure and antitumor activity. J Mar Biotechnol 1998, 6:208-213.

9. Kajiura H. Kakutani R, Akiyama T, Takata H, Kuriki T. A novel enzymatic process for glycogen production. Biocatal Biotransform 2008, 26:133-140. 
10. Kajiura H, Takata H, Kuriki T, Kitamura S. Structure and solution properties of enzymatically synthesized glycogen. Carbohydr Res 2010, 345:817-824.

11. Kajiura H, Takata H, Akiyama T, Kakutani R, Furuyashiki T, Kojima I, Harui T, et al. In vitro synthesis of glycogen: the structure, properties, and physiological function of enzymatically-synthesized glycogen. Biologia 2011, 66:387-394.

12. Kakutani R, Adachi Y, Kajiura H, Takata H, Kuriki T, Ohno N. Relationship between structure and immunostimulating activity of enzymatically synthesized glycogen. Carbohydr Res 2007, 342:2371-2379.

13. Kakutani R, Adachi Y, Kajiura H, Takata H, Kuriki T, Ohno N. Stimulation of macrophage by enzymatically synthesized glycogen: the relationship between structure and biological activity. Biocatal Biotransform 2008, 26:152-160.

14. Kakutani R, Adachi Y, Takata H, Kuriki T, Ohno N. Essential role of Toll-like receptor 2 in macrophage activation by glycogen. Glycobiol 2012, 22:146-159.

15. Kakutani R, Adachi Y, Kajiura H, Takata H, Kuriki T, Ohno N. The effect of orally administered glycogen on anti-tumor activity and natural killer cell activity in mice. Int Immunopharmacol 2012, 12:80-87.

16. Furuyashiki T, Takata T, Kojima I, Kuriki T, Fukuda I, Ashida H. Metabolic fate of orally administered enzymatically synthesized glycogen in rats. Food Funct 2011, 2:183-189.

17. Furuyashiki T, Ogawa R, Nakayama Y, Honda K, Kamisoyama H, Takata T, Yasuda M, et al. Enzymatically synthesized glycogen reduces lipid accumulation in diet-induced obese rats. Nutr. Res 2013, 33:743-752.

18. Savla GN, Palmer BW. Neuropsychology in Alzheimer's disease and other dementia research. Curr Opin Psychiatry 2005, 18, 621-627.

19. Falleti MG, Maruff P, Collie A, Darby D, McStephen M. Qualitative similarities in cognitive impairment associated with $24 \mathrm{~h}$ of sustained wakefulness and a blood alcohol concentration of $0.05 \%$. J Sleep Res 2003, 12:265-274.

20. Maruff P, Falleti MG, Collie A, Darby D, McStephen M. Fatigue-related impairment in the speed, accuracy and variability of psychomotor performance: comparison with blood alcohol levels. J Sleep Res 2005,14:21-27.

21. Katagiri M, Satoh A, Tsuji S, Shirasawa T. Effects of astaxanthin-rich Haematococcus pluvialis extract on cognitive function: a randomized, double-blind, placebo-controlled study. J Clin Biochem Nutr 2012, 51:102-107.

22. Collie A, Maruff P, Darby DG, McStephen M. The effects of practice on the cognitive test performance of neurologically normal individuals assessed at brief test-retest intervals. J Int Neuropsychol Soc 2003, 9:419-428. 
23. Pipingas A, Silberstein RB, Vitetta L, Rooy CV, Harris EV, Young JM, Frampton CM, et al. Improved cognitive performance after dietary supplementation with a Pinus radiata bark extract formulation. Phytother Res 2008, 22:1168-1174.

24. Terada Y, Fujii K, Takaha T, Okada S. Thermus aquaticus ATCC 33923 amylomaltase gene cloning and expression and enzyme characterization: production of cycloamylose. Appl Environ Microbiol 1999, 65:910-915.

25. Takata H, Ohdan K, Takaha T, Kuriki T, Okada S. Properties of branching enzyme from hyperthermophilic bacterium, Aquifex aeolicus, and its potential for production of highlybranched cyclic dextrin. J Appl Glycosci 2003, 50:15-20.

26. Takata H, Kajiura H, Furuyashiki T, Kakutani R, Kuriki T. Fine structural properties of natural and synthetic glycogens. Carbohydr Res 2009, 344:654-659.

27. Tafazoli S, Wong AW, Kajiura H, Kakutani R, Furuyashiki T, Takata H, Kuriki T. Safety evaluation of an enzymatically-synthesized glycogen (ESG). Regul Toxic Pharm 2010, 57:210-219.

28. Zhang Y, Moriguchi T, Saito H, Nishiyama N. Functional relationship between agerelated immunodeficiency and learning deterioration. Eur J Neurosci 1998, 10:3869-3875.

29. Lal H, Bennett M, Bennett D, Forster MJ, Nandy K. Learning deficits occur in young mice following transfer of immunity from senescent mice. Life Sci 1986, 39:507-512. 